\title{
Desde el ISET al InDRE. II. Instituto de Salubridad y Enfermedades Tropicales: madurez y consolidación, 1940-1964
}

Jorge Alejandro Ramírez-Hernández, ${ }^{1}$ María del Carmen Guzmán-Bracho, ${ }^{2 *}$ Carlos Viesca-Treviño ${ }^{1}$ y José Alberto Díaz-Quiñonez ${ }^{2}$

'Universidad Nacional Autónoma de México, Facultad de Medicina, Ciudad de México; ${ }^{2}$ Instituto de Diagnóstico y Referencia Epidemiológicos, Ciudad de México. México

\section{Resumen}

El periodo que comienza en 1940 es crucial para la creación del moderno sistema de salud de México. El Instituto de Salubridad y Enfermedades Tropicales es la institución pionera en investigación en salud y en sus primeras dos décadas logra madurez y consolida líneas de investigación expresadas en casi 1700 publicaciones. También obtiene notoria visibilidad internacional y fue seleccionado como referencia regional para la vigilancia epidemiológica de influenza, estreptococo y salmonela. Su evolución se produjo con ritmo de innovación que iría disminuyendo para el final de ese periodo. Enfrentó limitaciones para renovar líneas de trabajo, investigadores y equipo de laboratorio, sin embargo, su papel en las decisiones de la salud pública del país siguió siendo central.

PALABRAS CLAVE: Instituto de Salubridad y Enfermedades Tropicales. Historia de la medicina. Salud pública.

\section{Abstract}

The period that starts in 1940 is crucial for the creation of Mexico's modern health system. The Institute of Sanitary and Tropical Diseases is the pioneer institution in health research and on its first two decades it achieved maturity and consolidated lines of research expressed in almost 1700 publications. It also obtained notorious international visibility and was selected as a regional reference for epidemiological surveillance of influenza, streptococcus and salmonella. Its evolution took place with an innovation rhythm that would be decreasing by the end of this period. It faced limitations to renew lines of research, researchers and laboratory equipment. However, its role in public health decisions of the country remained central.

KEY WORDS: Institute of Public Health and Tropical Diseases. History of medicine. Public health.

Correspondencia:

*María del Carmen Guzmán-Bracho

E-mail: cguzmanbracho@ hotmail.com
Fecha de recepción: 12-04-2019

Fecha de aceptación: 02-05-2019

DOI: 10.24875/GMM.19005222
Gac Med Mex. 2019;155:391-398

Disponible en PubMed www.gacetamedicademexico.com 


\section{Introducción}

En 1935 comenzó la construcción del edificio del Instituto de Salubridad y Enfermedades Tropicales (ISET), en el predio denominado la Quinta de San Ramón (Figura 1).

El área donde se instaló había sido declarada de utilidad pública en 1934, para la construcción de diversas obras aprobadas por la $\mathrm{H}$. Comisión de Planificación del Distrito Federal en "beneficio de la salud pública", entre otras ganancias sociales intangibles propuestas en el decreto de expropiación de los predios conocidos con los nombres de Rancho Nextitla y Quinta de San Ramón. ${ }^{2}$

En esa zona, al noroeste de la ciudad de México, se inició entonces el periodo más importante de transformación del paisaje en la época posrevolucionaria. ${ }^{3}$ Se integró un polo educativo y de salud en un polígono que forman actualmente la Calzada México Tacuba, el Circuito Interior Instituto Técnico Industrial y la Calzada de Los Gallos. En el periodo 1946 a 1964 se incluyeron las instalaciones de la Unidad Profesional "Lázaro Cárdenas" del Instituto Politécnico Nacional (1936), ${ }^{1}$ el Hospital "Rubén Leñero" (1943), ${ }^{4}$ el Instituto de Virología, el Hospital de la Mujer (1955) y el que fuera la sede del ISET (ahora Instituto de Diagnóstico y Referencia Epidemiológicos) de 1935 a 2013.

El conjunto arquitectónico de la zona se comenzó a construir con el estilo propio de los años treinta y cuarenta del siglo XX; en la Escuela Normal Superior y el ISET se identifican huellas de la Bauhaus y art déco europeos.

El nombre del Instituto estuvo constituido por tres términos:

- Instituto (institūtum, 'regla”, 'enseñanza'), término previamente utilizado en la salud en México, como en el Instituto Médico Nacional, Instituto Patológico, Instituto Bacteriológico/Instituto de Higiene, a la manera de las instituciones europeas y norteamericanas. Por lo tanto, se perfiló una institución dedicada a la investigación.

- Salubridad (salubritas, 'buena salud general') es un concepto clave en la salud de México a partir del Consejo Superior de Salubridad, órgano rector en esta área desde 1841 y reformado en 1917 como Departamento de Salubridad Pública y Consejo de Salubridad General. ${ }^{6}$

- Enfermedades tropicales, es una noción de cuño colonial de finales del siglo XIX, que reconocía la asociación entre ambiente geográfico y estado

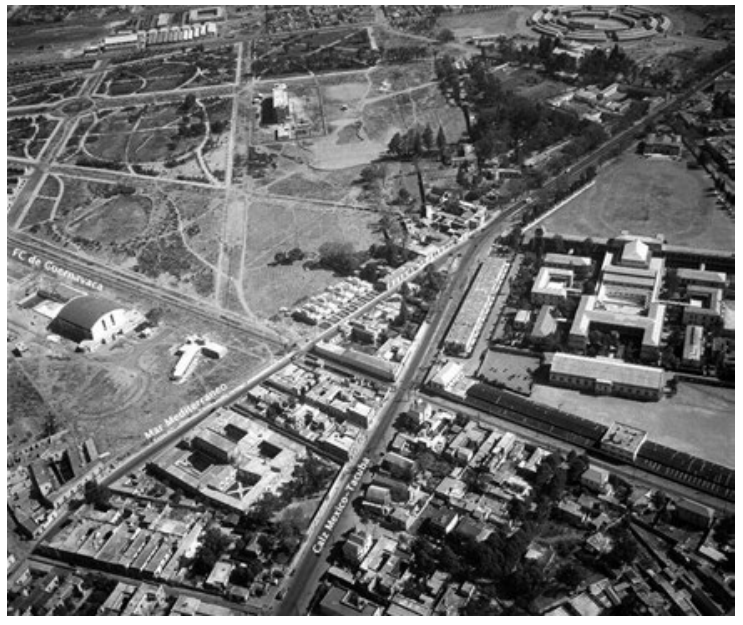

Figura 1. El ISET en construcción, 1935. Es un edificio único, en un espacio destinado a la salud. Foto disponible en línea. Original perteneciente al Fondo Aerofotográfico de ICA.

de salud de una población. El paludismo, la leishmaniasis, la oncocercosis y la enfermedad de Chagas son ejemplo de enfermedades tropicales con impacto epidemiológico en México.

En el periodo de 1940 a 1964, las enfermedades infecciosas fueron la principal causa de muerte. La tosferina y el paludismo constituyeron una causa importante de morbimortalidad en la primera mitad del siglo $X X$, además de las afecciones gastrointestinales y las de vías respiratorias. Sin embargo, la distribución y aplicación de la vacuna antivariolosa, la introducción del uso clínico de la penicilina y otros antibióticos, la aplicación del DDT como plaguicida, además de las medidas de saneamiento básico implementadas, de alimentación y legislación ${ }^{8}$, modificaron el patrón de las enfermedades causantes de muerte en la población ${ }^{9}$ (Tabla 1).

\section{Segundo plan sexenal}

En el sexenio que comenzó en 1940 se desarrolló una intensa actividad de diseño y construcción de infraestructura por parte de la Secretaría de Asistencia, así como de legislación que llevaría a crear el Instituto Mexicano del Seguro Social. En el informe de labores de Gustavo Baz, ${ }^{10}$ Secretario de Asistencia Pública, presentado al H. Ejecutivo de la Unión para el periodo 1941-1942, se delineó el trabajo de las comisiones técnicas hospitalarias y sus planes de construcción y unidades de asistencia. El modelo de hospital general del que se partió fue el hospital vertical a la manera de los que existían en Estados Unidos, en el que se ofrecían servicios clínicos básicos, con laboratorio y archivo, además de constituir un centro 
Tabla 1. Quince principales causas de muerte en México $^{9}$

\begin{tabular}{|c|c|c|c|c|}
\hline Núm. & 1930 & 1940 & 1950 & 1960 \\
\hline 1 & Diarrea y enteritis & Diarrea y enteritis & $\begin{array}{l}\text { Gastritis, duodenitis, enteritis y } \\
\text { colitis, excepto diarrea en el recién } \\
\text { nacido. }\end{array}$ & $\begin{array}{l}\text { Gastritis, duodenitis, enteritis } \\
\text { y colitis, excepto diarrea en el } \\
\text { recién nacido }\end{array}$ \\
\hline 2 & Influenza y neumonías & Influenza y neumonías & Influenza y neumonías & Influenza y neumonías \\
\hline 3 & Paludismo & Paludismo & $\begin{array}{l}\text { Enfermedades de la primera } \\
\text { infancia }\end{array}$ & $\begin{array}{l}\text { Enfermedades de la primera } \\
\text { infancia }\end{array}$ \\
\hline 4 & tos ferina & $\begin{array}{l}\text { Accidentes o muertes } \\
\text { violentas }\end{array}$ & Accidentes o muertes violentas & Accidentes o muertes violentas \\
\hline 5 & $\begin{array}{l}\text { Accidentes o muertes } \\
\text { violentas }\end{array}$ & $\begin{array}{l}\text { Deformaciones congénitas y } \\
\text { enfermedades de la primera } \\
\text { infancia }\end{array}$ & Paludismo & Enfermedades del corazón \\
\hline 6 & Viruela & Sarampión & Enfermedades del corazón & Tumores malignos \\
\hline 7 & Sarampión & Bronquítis & tos ferina & Bronquítis \\
\hline 8 & $\begin{array}{l}\text { Debilidad congénita, } \\
\text { vicios de conformación } \\
\text { congénita }\end{array}$ & $\begin{array}{l}\text { Enfermedades del hígado y } \\
\text { de las vías biliares }\end{array}$ & Tuberculosis TF & Tuberculosis TF \\
\hline 9 & Tuberculosis TF & Tuberculosis TF & Bronquítis & Cirrosis hepática \\
\hline 10 & $\begin{array}{l}\text { Enfermedades del hígado } \\
\text { y de las vías biliares }\end{array}$ & Enfermedades del corazón & Sarampión & Paludismo \\
\hline 11 & Bronquítis & Tos ferina & Tumores malignos & $\begin{array}{l}\text { Lesiones vasculares que } \\
\text { afectan al sistema nervioso } \\
\text { central }\end{array}$ \\
\hline 12 & $\begin{array}{l}\text { Enfermedades del } \\
\text { corazón }\end{array}$ & Tifoidea y paratifoidea & Cirrosis del hígado & Sarampión \\
\hline 13 & Tifoidea y paratifoidea & Nefritis & Nefritis y nefrosis & Disentería TF \\
\hline 14 & Nefritis & Tumores malignos & Disentería TF & Tos ferina \\
\hline 15 & $\begin{array}{l}\text { Hemorragia cerebral, } \\
\text { embolia o trombosis }\end{array}$ & $\begin{array}{l}\text { Hemorragia cerebral, embolia } \\
\text { o trombosis }\end{array}$ & Tifoidea & Anemia \\
\hline
\end{tabular}

$\mathrm{TF}=$ Todas las formas.

docente. Todo ello en la fórmula "el hospital es al mismo tiempo un centro científico, un hotel, una planta industrial y una escuela". ${ }^{10}$ En esa época estaban en construcción hospitales generales en todo el país, el Instituto Nacional de Cardiología y el Hospital Infantil. Se estaban adquiriendo los terrenos para la construcción del Centro Médico Nacional en la colonia Doctores, en la Ciudad de México. ${ }^{6}$

\section{Un nuevo modelo hospitalario y de instituto de investigación}

El Plan de Hospitales, concebido por Gustavo Baz, define los elementos clave que debe tener el establecimiento hospitalario. Surgen varios hospitales e institutos de especialidad que van a competir para ser líderes de esta naciente red de atención a la salud.

Con el Instituto Nacional de Cardiología se establecieron tres puntos nuevos en su relación con la Secretaría de Salubridad y Asistencia: ${ }^{11}$

- Cierta autonomía de gobierno.

- Una parte del servicio podía ser utilizado por pacientes privados.

- Su situación legislativa permitía la recepción de donativos.

En 1943 se produjeron dos hechos fundamentales para la salud del país:

- La Secretaría de Asistencia incorporó al Departamento de Salubridad Pública para transformarse en la Secretaría de Salubridad y Asistencia (SSA).

- Se aprobó la legislación para la creación del Instituto Mexicano del Seguro Social, que inició funciones en $1944 .^{12,13}$ 
De esta manera, Gustavo Baz, primer secretario de Salubridad y Asistencia, se convirtió en el más influyente médico mexicano de la era posrevolucionaria. Probablemente ningún país en proceso de industrialización en el mundo, durante la guerra, emprendió un esfuerzo comparable para la atención de la salud. Con este ímpetu creativo en México, los nuevos institutos de salud se crearon bajo criterios distintos a los del ISET.

\section{¿Dónde se ubicó el ISET en este periodo?}

Por un lado, el ISET no tenía autonomía y se regía con la legislación general de la SSA, con financiamiento rígido y mínimas cuotas de recuperación de sus servicios clínicos y de laboratorio. Debido a ello, el Instituto se iría quedando en desventaja para competir e impulsar la investigación innovadora, atraer a nuevas generaciones de investigadores y renovar el equipo y mobiliario de laboratorio.

\section{Cambios en la economía mexicana}

La economía mexicana se transformó durante el sexenio de Manuel Ávila Camacho. ${ }^{12,13}$ Se aplicó una política de estímulo y apoyo al sector privado, que había tenido desconfianza en el sexenio de Lázaro Cárdenas, lo que permitió el regreso de la inversión privada al país. ${ }^{12}$

En 1941, Estados Unidos ingresó a la guerra y flexibilizó su trato con México, endurecido a consecuencia de la nacionalización petrolera. Estados Unidos le concedió a México préstamos para desarrollar su industria e incrementó las importaciones desde nuestro país. Por su parte, el Estado mexicano ofreció cinco años sin impuestos a las nuevas industrias como incentivo para su impulso. El Banco de México efectuó la regulación oficial y la Nacional Financiera amplió créditos. Surgieron empresas nacionales en las ramas de acero, vidrio, cemento, guanos y fertilizantes; se fortaleció el sector ferroviario y la industria petrolera, entre otros. ${ }^{8,12}$

Lo anterior, aunado a la limitación que la Guerra Mundial produjo en las exportaciones desde Europa hacia Estados Unidos, permitió que México empezara a llenar ese lugar y el Estado apoyara decididamente la industrialización del país. Así se generó un efecto de arrastre de la economía norteamericana sobre la mexicana, con el consiguiente crecimiento económico, lo cual permitió expandir la infraestructura de salud.
Los trabajadores de la nueva industria nacional obtuvieron atención a su salud en el Instituto Mexicano del Seguro Social, aliviando de peso económico a la SSA.

\section{Impulso a la investigación en el ISET}

Se dispone de claros ejemplos del alcance internacional del ISET a través de su presencia en la investigación y en las políticas de salud. En 1946, Luis Mazzotti efectuó un viaje de estudio de seis meses por nueve países de África y Medio Oriente. ${ }^{14} \mathrm{Su}$ finalidad era conocer in situ cómo se enfrentaban los problemas de algunas enfermedades infecciosas desde los centros de investigación y hospitales en las colonias europeas de Egipto, África árabe (Túnez, Argelia), África negra (Sudán, Uganda, Kenia, Congo Belga, África ecuatorial) y Sudáfrica. Mazzotti tuvo acceso a interesantes estudios sobre virus - no publicados en esa fecha- y recibió instrucción en cultivo de parásitos y artrópodos, identificación clínica y diagnóstico por laboratorio de enfermedades como tifo, tripanosomiasis, leishmaniasis visceral, esquistosomiasis, sífilis; revisó con detalle las estrategias de eliminación y campañas contra Anopheles, fiebre amarilla, uncinariasis, esquistosomiasis, oncocercosis $y$, en particular, sobre saneamiento ambiental y rural. Fue un viaje distinto a los acostumbrados entonces por médicos de su generación, que asistían a centros académicos de Europa y Estados Unidos. En su visita, Luis Mazzotti observó la situación en los países de menor ingreso del mundo y analizó las estrategias que los países colonialistas empleaban ante las enfermedades infecciosas.

En otro extremo de la actividad internacional del ISET, el Consejo Económico y Social de Naciones Unidas organizó en 1946 una Conferencia Internacional de Salud para proponer una organización internacional de salud dependiente de las Naciones Unidas, en reconocimiento de la urgente necesidad de acción internacional en el ámbito de la salud pública. Se invitó a algunos países miembros para enviar expertos en salud pública. México estuvo representado por Manuel Martínez Báez, entonces Subsecretario de Salubridad y Asistencia, quien poco antes había sido director del ISET (Figura 2); fue miembro del Comité Técnico Preparatorio de la constitución de la Organización Mundial de la Salud. ${ }^{15}$

Ambas actividades internacionales constituyen ejemplo de dos perfiles institucionales, local y global. 
“(3) establishes a Technical Preparatory Committee to prepare a draft annotated agenda and proposals for the consideration of the Conference, and appoints the following experts or their alternates to constitute the Committee :

I. Dr. Gregorio Berman (Argentina)

2. Dr. René SAND (Belgium)

3. Dr. Geraldo H. DE Paula Souza (Brazil)

4. Major-General G. B. Chrsholm (Canada)

5. Dr. P. Z. KING (China) (alternate: Dr. Szeming Sze)

6. Dr. Josef ČAnčrk (Czechoslovakia)

7. Dr. Aly Tewfik Shousha Pasha (Egypt)

8. Dr. A. Cavaillon (France) (alternate: Dr. Leclainche)

9. Dr. KOPANARIS (Greece)

ro. Major C. Mani (India) (alternate: Dr. Chuni LAL Katial)

II. Dr. Manuel Martinez Baez (Mexico)

12. Dr. Karl Evang (Norway)

13. Dr. Martin KACPRZAK (Poland)

14. Sir Wilson Jameson (United Kingdom) (alternate: Dr. Melville MACKENZIE)

15. Surgeon-General Thomas PARRAN (United States of America) (alternate: Dr. James A. Doull)

I6. Dr. Andrija Stampar (Yugoslavia)

and, in a consultative capacity, representatives of :

Figura 2. Constitución de la Organización Mundial de la Salud. Comité Técnico Preparatorio. El Consejo Económico y Social de las Naciones Unidas, derivado de una resolución fechada el 15 de febrero de 1946, convocó a la Conferencia Internacional de la Salud, para el establecimiento de la Organización Mundial de la Salud.
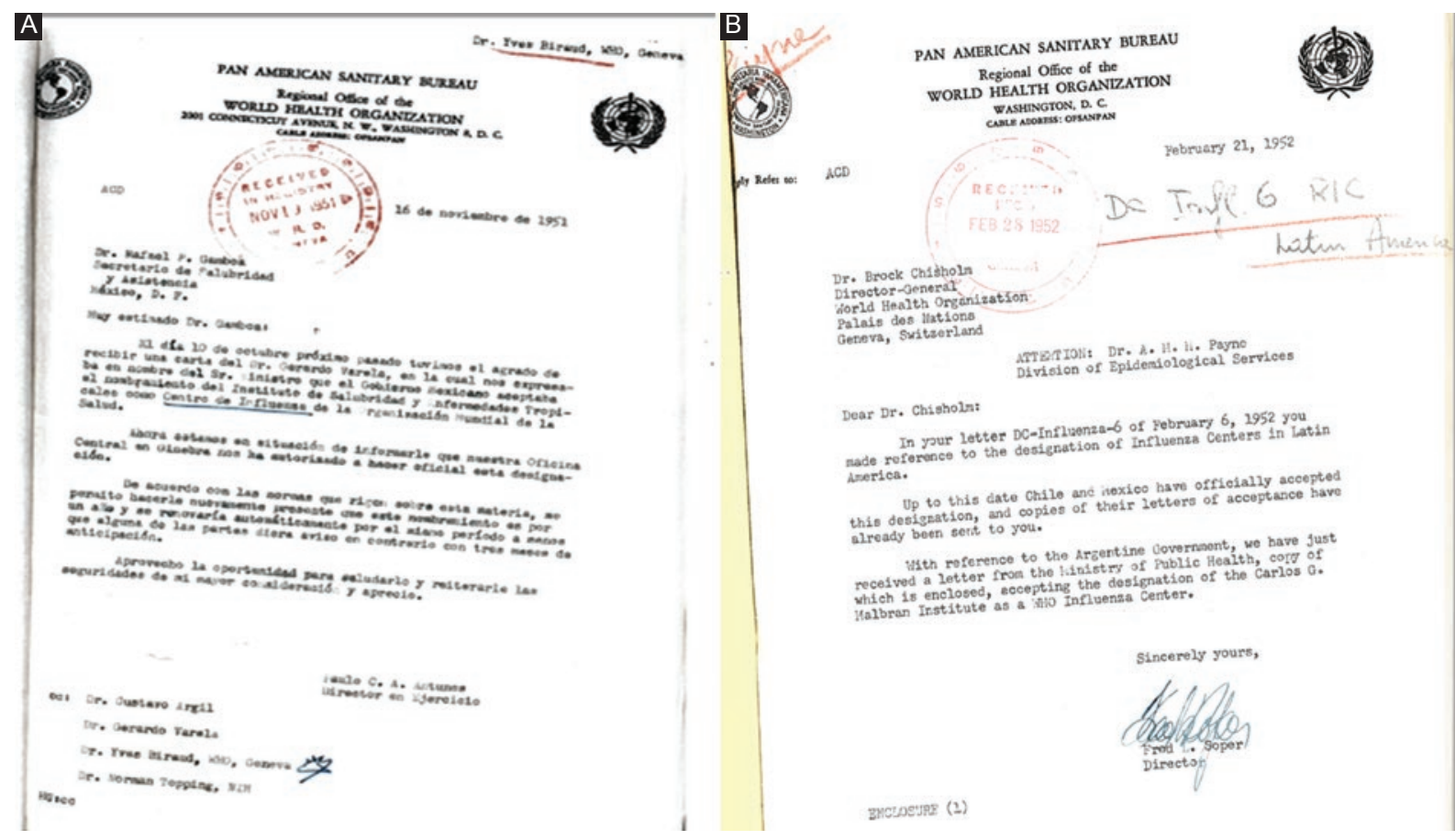

Figura 3. ISET, Centro de Influenza en Latinoamérica. A) Designación del ISET, 1951. B) Notificación de la designación a la Organización Mundial de la Salud, 1952.

La importante labor científica del ISET se manifestó también en varias áreas de alta especialidad creadas en la época. En 1951, el director de la Organización Sanitaria Panamericana incorporó a México junto con Chile, en Latinoamérica, en la vigilancia goblal de influenza. EI ISET se convirtió en el primer Centro Nacional de Influenza en Latinoamérica, un año antes de la creación del Global Influenza Surveillance and Response System que integró a los Centros Nacionales de Influenza con el propósito de monitorear la circulación de los virus, identificar su potencial pandémico y actualizar la composición anual de la vacuna (Figura 3).

Otras instancias del ISET reconocidas fueron el Centro de Salmonellas en México y el Centro para la 
Tipificación de Estreptococos, que tuvieron responsabilidad regional. ${ }^{16,17}$ Un trabajo de gran importancia se llevó a cabo en el Laboratorio de Micología y la Clínica de Dermatología Tropical, con la investigación de los agentes etiológicos y la revisión diaria de casos clínicos a cargo de Antonio González Ochoa (C. Contreras, comunicación personal), considerado el iniciador de la investigación en micología médica en México, con el respaldo de publicaciones de impacto mundial.

En 1954 se presentó el proyecto para establecer un laboratorio específico para virología, si bien desde principios de esa década en el Instituto se realizaba cultivo de tejidos, aislamiento y reconocimiento serológico viral (enterovirus, poliovirus), mantenimiento de cepas de poliovirus, así como pruebas diagnósticas y aislamiento del virus rábico. ${ }^{18}$

\section{Dirección del Instituto}

Esta exitosa institución en sus inicios fue dirigida por un consejo directivo integrado por los investigadores jefes de laboratorio y presidido por el director. ${ }^{19} \mathrm{En}$ los 24 años de recorrido que comprende este artículo, el ISET estuvo dirigido por sus cuatro principales investigadores fundadores y José Zozaya ${ }^{20}$ (Tabla 2).

\section{Publicaciones nacionales e internacionales $^{21}$}

A partir del primer año de trabajo del ISET se inició la publicación de una revista propia, que apareció regularmente con el nombre de Revista del Instituto de Salubridad y Enfermedades Tropicales (Revista ISET), de noviembre de 1939 a diciembre de 1965, cuando cambió de nombre a Revista de Investigación en Salud Pública con la indicación "Antigua Revista del Instituto de Salubridad y Enfermedades Tropicales". ${ }^{22}$ La Revista ISET publicó parte del profuso trabajo de los investigadores del Instituto. Hasta 1964 se tenían 702 artículos de 266 autores, en los que se identifica la participación de investigadores internacionales, huéspedes temporales de la institución, como Robert Hegner, Herman Mooser, Emile Brump, Ernest Faust, Todd Hewitt y Norman Stoll, y la colaboración de miembros del ISET con investigadores nacionales de otras dependencias. Entre 1939 a 1964, Luis Vargas, Gerardo Varela y Luis Mazzotti publicaron más de un centenar de artículos cada uno, con un promedio de cinco anuales, solo en la Revista ISET (Tabla 3). Lo publicado en esta revista y en 97
Tabla 2. Directores del Instituto de Salubridad y Enfermedades Tropicales de 1938 a $1964^{a, b}$

\begin{tabular}{|c|c|}
\hline Director & Periodo \\
\hline Eliseo Ramírez Ulloa & 1938 \\
\hline Manuel Martínez Báez & $1939-1940$ \\
\hline Eliseo Ramírez Ulloa & 1940 \\
\hline Manuel Martínez Báez & 1941 \\
\hline Miguel E. Bustamante Vasconcelos & $1941-1943$ \\
\hline José Zozaya Stille & 1943-1946 \\
\hline Miguel E. Bustamante Vasconcelos & $1946-1947$ \\
\hline Gerardo Ignacio Varela Mariscal & $1948-1952$ \\
\hline Manuel Martínez Báez & 1952-1959 \\
\hline Gerardo Ignacio Varela Mariscal* & 1959-1977 \\
\hline \multicolumn{2}{|c|}{$\begin{array}{l}\text { *Antonio González Ochoa fue director interino de 1975-1977. } \\
\text { aOrganización Sanitaria Panamericana. Boletín de la Oficina Sanitaria Panamericana 1938; } 269 \\
\text { bValdespino-Gómez JL, García-García ML, Del-Río-Zolezzi A, et al. El Instituto Nacional } \\
\text { de Diagnóstico y Referencia Epidemiológicos “Dr. Manuel Martínez Báez" en } 1994 . \\
\text { p. 189-197. En: Una institución académica y dieciséis investigadores distinguidos. } \\
\text { Quincuagésimo quinto aniversario del INDRE. Secretaría de Salud. México. } 1994 .\end{array}$} \\
\hline
\end{tabular}

más, abarca diversas áreas de interés en salud pública como la geografía médica, epidemiología, aspectos inmunológicos, comportamiento clínico, estrategias de tratamiento, biología y susceptibilidad a antimicrobianos, taxonomía y control de vectores. ${ }^{23}$

Los resultados reportados ${ }^{16,17,24}$ son producto de:

- Actividades experimentales en el laboratorio y con los datos obtenidos en el procesamiento de muestras mediante diversas pruebas analíticas.

- El intenso trabajo en la clínica y tratamiento de enfermedades tropicales y en pacientes con micosis profundas y dermatológicas.

- El estrecho trabajo colaborativo con las Campañas Nacionales y "programas aplicativos" (enfermedades transmitidas por vector, pinto, brucelosis, diarreas y enfermedades propias de la infancia, poliomielitis, enfermedades venéreas, entre otras).

- Estudios especiales en zonas de riesgo, endémicas 0 epidémicas, en todo el país (enfermedad de Chagas, oncocercosis, paludismo, histoplasmosis, leishmaniasis, rickettsiosis, helmintiasis, entre otras)

\section{Conclusiones}

En el periodo 1940 a 1964, el ISET fue la institución pionera de la investigación interdisciplinaria en salud en México, desarrollando aspectos básicos, clínicos, entomológicos, epidemiológicos y con repercusión en 
Tabla 3. Artículos publicados por investigadores del Instituto de Salubridad y Enfermedades Tropicales entre 1939 y $1964^{2}$

\begin{tabular}{|c|c|c|c|c|}
\hline & Autor & $\begin{array}{c}\text { Revista } \\
\text { ISET }\end{array}$ & $\begin{array}{c}\text { Otras } \\
\text { revistas* }\end{array}$ & Tota \\
\hline 1 & L. Vargas & 117 & 130 & 247 \\
\hline 2 & E. Roch & 13 & 228 & 241 \\
\hline 3 & G. Varela & 101 & 82 & 183 \\
\hline 4 & L. Mazzotti & 100 & 76 & 176 \\
\hline 5 & A. González Ochoa & 52 & 71 & 123 \\
\hline 6 & E. Beltrán & 48 & 62 & 110 \\
\hline 7 & M. Martínez Báez & 17 & 86 & 103 \\
\hline 8 & A. P. León & 41 & 33 & 74 \\
\hline 9 & E. C. Del Pozo & 6 & 67 & 73 \\
\hline 10 & M. E. Bustamante & 28 & 26 & 54 \\
\hline 11 & A. Díaz Nájera & 37 & 9 & 46 \\
\hline 12 & M. González Rivera & 7 & 31 & 38 \\
\hline 13 & M. A.Bravo Becherelle & 15 & 21 & 36 \\
\hline 14 & J. Zozaya & 26 & 3 & 29 \\
\hline 15 & S. C. Campillo & 9 & 17 & 26 \\
\hline
\end{tabular}

*Se incluyen 97 distintas revistas, capítulos de libro, boletines, etcétera, nacionales e internacionales

las campañas sanitarias del país. Logró un impulso y productividad extraordinarios en sus primeras décadas de trabajo para comenzar a disminuir su ritmo de innovación, actualización de personal y equipos de laboratorio. A partir de 1943 surgieron otras instituciones de investigación en salud con acuerdos institucionales distintos al del ISET, con mayor autonomía y capacidad de gestión de recursos. Además, en esa coyuntura de crecimiento económico del país, se creó la Secretaría de Salubridad y Asistencia, así como el Instituto Mexicano del Seguro Social; la primera produjo el Plan de Hospitales y se volvió imperioso disponer de un modelo hospitalario y de un discurso adecuado para motivar y encauzar la formación y reproducción del saber médico, de enfermería y de otras profesiones de la salud. Los nacientes institutos también ofrecieron atractivas carreras clínicas con potencial de mayor ingreso que la investigación de tiempo completo en temas de salubridad y enfermedades tropicales.

Sin embargo, el ISET multiplicó sus actividades y se volvió central en la toma de decisiones importantes en la salud pública del país en relación con los problemas de tipo infeccioso. Ganó notoriedad internacional y obtuvo nombramientos regionales formales, como sucedió en influenza, salmonela y estreptococos.
En una interesante aportación, el médico e historiador Fernando Martínez Cortés analiza el nacimiento, vocación y liderazgo tanto del ISET como del Instituto Nacional de Cardiología: 25

[EI ISET] ...nace con el expreso fin de contribuir a los problemas de salubridad que aquejan al país, entre las cuales las llamadas enfermedades tropicales constituyen una parte importante. Podemos decir que dicha institución es la respuesta a una ideología, a un proyecto político orientado a mejorar la salud de los mexicanos. En cambio, el Instituto Nacional de Cardiología, que inaugura el presidente Manuel Ávila Camacho, nace de una especialidad médica eminentemente curativa y se crea para investigar y para enseñar, con los medios más adelantados los problemas de esa especialidad... sin que las cardiopatías constituyeran un gran problema de salud a nivel nacional, al lado de la personalidad de Chávez se unieron el apoyo oficial y el privado.

EI ISET alcanzó un gran punto de madurez, consolidación institucional y reconocimiento internacional en este periodo.

\section{Agradecimientos}

Al Fondo Aerofotográfico de la Fundación ICA, por la validación de la pertenencia del original de foto del ISET en construcción de 1935, así como por la validación de la fecha de toma. A las autoridades y personal del Archivo Histórico de la Secretaría de Salud, por compartir desinteresadamente sus conocimientos y por su valiosa orientación en la revisión de los expedientes. A José Luis Garnica Carreño, Library and Information Networks for Knowledge, por la asistencia proporcionada en la sede de la Organización Mundial de la Salud en Ginebra, Suiza.

\section{Bibliografía}

1. Calvillo-Velasco M, Valencia-Flores AO. El Cuadrilátero, recinto histórico. La formación de un ícono de identidad del Instituto Politécnico Nacional (1922-1914). México: Instituto Politécnico Nacional; 2015.

2. Decreto que declara de utilidad pública la expropiación de los predios Rancho Nextitla y Quinta de San Ramón, ubicados en la ex-municipalidad de Tacuba de Morelos. México: Diario Oficial de la Federación; 1934.

3. Molina-Palestina O. Breve historia y relación del patrimonio tangible de la delegación Miguel Hidalgo. México: Delegación Miguel Hidalgo; 2012.

4. Fajardo-Ortiz G. Dr. Rubén Leñero Ruiz (1902-1942): un hospital lleva su nombre. Bol Mex Hist Fil Med. 2002;5:27-28.

5. Secretaría de Salud. Historia del Hospital de la Mujer. México: Secretaría de Salud. 2018.

6. Bustamante ME, Viesca-Treviño C, Villaseñor F, Vargas-Flores A, Castañón R, Martínez-Barbosa X. La salud Pública en México. 1959-1982. México: Secretaría de Salubridad y Asistencia; 1982

7. Fernández-De Castro J, Fernández-De Castro H. Epidemias y endemias de México en el siglo XX. México: Siglo XXI Editores; 2015.

8. Ávila-Camacho M. Primer informe de gobierno. México: Congreso de la Unión; 1941.

9. Almada-Bay I. La mortalidad en México. Colección Salud y Seguridad Social. Serie Manuales Básicos y Estudios. México: Instituto Mexicano del Seguro Social; 1982

10. Baz G. Informe de Labores presentado al H. Ejecutivo de la Unión. México: Secretaría de Asistencia Pública; 1941-1942.

11. Chávez-Rivera I. Instituto Nacional de Cardiología Ignacio Chávez. Cinco décadas de historia. 1944-1994. México: Editorial Médica Panamericana; 1999 
12. IMSS. El Instituto Mexicano del Seguro Social. México: IMSS; 1943.

13. Zertuche-Muñoz, F. Historia del Instituto Mexicano del Seguro Social. Los primeros años 1943-1944. México: Instituto Mexicano del Seguro Social; 1980

14. Archivo Histórico de la Secretaría de Salud. Fondo ISET SSA/SUBSyA 02/073-33/1

15. World Health Organization. Official records of the World Health Organization No. 2. Summary report on proceedings, minutes and final acts of the International Health Conference Held in New York from 19 june to 22 july 1946. EE. UU: United Nations/World Health Organization; 1948.

16. González-Rivera M. Instituto de Salubridad y Enfermedades Tropicales de México: diez años de trabajo. Bol Ofic Sanitaria Panam. 1949;28: 702-718.

17. Varela G. Instituto de Salubridad y Enfermedades Tropicales. Salud Publica Mex. 1964;6:1165-1171.

18. Archivo Histórico de la Secretaria de Salud. Fondo SSA, SUBSA C66E1.
19. Martínez-Báez M. El Instituto de Salubridad y Enfermedades Tropicales, 1969. An Soc Hist Cienc Tecnol. 1969;1:143-162.

20. Ramírez-Hernández JA, Guzmán-Bracho MC, Díaz-Quiñonez JA. Desde el ISET al InDRE. I. Instituto de Salubridad y Enfermedades Tropicales: génesis y primeros años, 1934-1940. Gac Med Mex. 2019;155(3):322-327.

21. Colección de la Revista del Instituto de Salubridad y Enfermedades Tropicales del Instituto de Diagnóstico y Referencia Epidemiológicos. México: Instituto de Diagnóstico y Referencia Epidemiológicos.

22. Marcial LD. Las publicaciones oficiales de la Secretaria de Salubridad y Asistencia. Salud Publica Mex. 1983;25:489-497.

23. Instituto de Salubridad y Enfermedades Tropicales. Monografía No. 4 Bibliografía General 1939-1964. Rev Inst Salubridad Enferm Trop. 1964;24:1-151

24. Mazzotti L. Dirección General de Institutos y Laboratorios. Salud Publica Mex. 1964:6:127-132.

25. Martínez-Cortés F. Introducción. En: Bustamante ME. Cinco personajes de la Salud en México. México: Porrúa; 1986. 